\title{
Erratum to: Introduction to the theme issue on variability modeling of software-intensive systems
}

\author{
Andrzej Wąsowski ${ }^{1}$ - Thorsten Weyer ${ }^{2}$
}

Published online: 16 November 2015

(C) Springer-Verlag Berlin Heidelberg 2015

\section{Erratum to: Softw Syst Model}

DOI 10.1007/s10270-015-0501-1

Unfortunately there is a mistake in the author information for references $[1,5]$ in the text.

The correct information is (as in the references itself):

"Bonifácio, Borba, Ferraz, and Accioly ('Empirical assessment of two approaches for specifying software product lines use case scenarios' [5]) report the results of an empirical investigation of two different specification approaches for software product line scenarios, ..." and "In 'The shape of feature code: an investigation of twenty c-preprocesor-based system' [1] Queiroz, Passos, Valente, Hunsen, Apel, and Czarnecki investigate twenty projects using C preprocessor directives for variability management."

The online version of the original article can be found under doi:10.1007/s10270-015-0501-1.

$\triangle$ Andrzej Wąsowski

wasowski@itu.dk

1 IT University of Copenhagen, Copenhagen, Denmark

2 University of Duisburg-Essen, Essen, Germany 